\title{
Carbon dot as fluorescent probe for detection of chromium(VI)*
}

\author{
Li-Yang Fang, Jing-Tang Zheng ${ }^{\dagger}$ and Zhen-Zhen Chen \\ State Key Laboratory of Heavy Oil Processing, China University of Petroleum, \\ Qingdao 266580, Shandong, PR China \\ 'E-mail: jtzheng03@163.com
}

\begin{abstract}
Hexavalent chromium $(\mathrm{Cr}(\mathrm{VI}))$ is a well known toxic carcinogens. Therefore, the convenient and quick detection of $\mathrm{Cr}(\mathrm{VI})$ in the environment is highly desired . In this study, a new method for determination of $\mathrm{Cr}(\mathrm{VI})$ was developed based on its quenching effect on the fluorescent of carbon quantum dot (C-dots). The fluorescent C-dots with strong photoluminescence and high photostability were prepared via a low cost, facile one-step hydrothermal approach from ammonium citrate. The C-dots probe shows many obvious advantages of convenience and simplicity. The C-dots-based sensor has highly selectivity and sensitive for $\mathrm{Cr}(\mathrm{VI})$ in a wide concentration range of $0-50 \mu \mathrm{M}$. The detection limitation comes to $0.015 \mu \mathrm{M}$.
\end{abstract}

Keywords: Ammonium Citrate Derived Carbon Dots, Fluorescent Probe, The Detection Of $\mathrm{Cr}(\mathrm{VI})$.

\section{Introduction}

Hexavalent chromium $(\mathrm{Cr}(\mathrm{VI}))$ has highly toxic and carcinogenic properties. The wide industrial application and other anthropogenic processes have been inevitably releasing chromium ions into the environment, leading to ecological damage from chromium(VI) contamination. The detection of chromium(VI) becomes very important and imperative in environmental samples. The traditional chromium(VI) analysis of detection means are time-consuming, complicated or expensive cost due to complicated sample pretreatment and the requirement of expensive equipment[1-3]. Therefore, it is very urgently needed for trace $\mathrm{Cr}(\mathrm{VI})$ detection to develop much simple, convenient and quick analysis techniques.

In recent years, the fluorescence probes have been successfully applied as fluorescent sensor because of the easy operation, high sensitivity and selectivity. Compared with semiconductor quantum dots and organic dyes, carbon quantum dots (C-dots) are not only electron donor but also electron acceptor and possess

\footnotetext{
* This work is financially supported by the National Natural Science Foundation of China (No.
} 21376268) 
many advantages such as low and non-toxicity, excellent biocompatibility, good chemical and photo stability and tunable excitation and emission features[4-6], which made C-dots as a good alternative to be used as fluorescent sensors for the detection of $\mathrm{pH}$, molecular substances and ions. The previously reported $\mathrm{C}$ dots-based fluorescent probe for the determination of $\mathrm{Cr}(\mathrm{VI})$ are relatively few. Consequently, wide room is left to study how to obtain C-dots probe with high sensitivity and selectivity for $\mathrm{Cr}(\mathrm{VI})$.

In the work, we first synthesized high fluorescence C-dots via a low cost, facile one-step hydrothermal approach from ammonium citrate. These carbon dots show strong fluorescence emission $(\mathrm{QY}=18.95 \%)$ which benefits for the $\mathrm{Cr}(\mathrm{VI})$ detection. The structures, optical properties and photostability of the asprepared C-dots were studied. In particular, fluorescence responses of the asprepared C-dots for some kinds of metal ions were investigated. Based on the fluorescence quenching for $\mathrm{Cr}(\mathrm{VI})$, the as-made $\mathrm{C}$-dots were applied as fluorescent probe for the determination of $\mathrm{Cr}(\mathrm{VI})$. The C-dots-based probe show a series of merits such as good selectivity and highly sensitive. The detection limitation is $0.015 \mu \mathrm{M}$. Therefore, the as-prepared C-dots-based fluorescence probe can be have potential application for the determination of chromium $(\mathrm{Cr}(\mathrm{VI}))$ in the environmental industry.

\section{Experimental Section}

\subsection{Materials}

All reagents(AR) were purchased from commercial suppliers (Sinopharm Chemical Reagent Co., Ltd) and used without further purification. Deionized water (distilled) was used for the preparation of all aqueous solutions. All metal ions solutions were made from their chloride, sulfate, or nitrate salts. $\mathrm{Cr}$ (VI) stock solution $\left(1.00 \times 10^{-3} \mathrm{M}\right)$ was prepared by dissolving $29.4 \mathrm{mg} \mathrm{K}_{2} \mathrm{Cr}_{2} \mathrm{O}_{7}$ in $100 \mathrm{~mL}$ water and stored in dark. The original stock solution can be diluted to different concentrations and used in the analytical procedure.

\subsection{Synthesis of C-dots}

Ammonium citrate solution $(0.2 \mathrm{M})$ was heated at $180^{\circ} \mathrm{C}$ for 4 hour in teflonlined stainless-steel autoclave, then cooled down to room temperature. Purification of the samples was completed through centrifuged and filter. The obtained samples were then concentrated and dried. Finally, the C-dots were obtained and recorded as CDs- 180 . 


\subsection{Detection test}

C-dots solution $(0.2 \mathrm{~g} / \mathrm{L})$ was prepared by dissolving C-dots in deionized water. Then different volume of $\mathrm{Cr}(\mathrm{VI})$ aqueous solution $\left(1 \times 10^{-5} \mathrm{M}\right)$ was added stepwise into the $\mathrm{C}$-dots solution to form mixed solution in which $\mathrm{Cr}(\mathrm{VI})$ concentration range is from 0 to $50 \mu \mathrm{M}$ with a $2 \mu \mathrm{M}$ spacing, respectively. After that, the fluorescence emission spectra of the mixed solution were measured.

\section{Results and Discussion}

\subsection{Structural of the C-dots}

The morphology and structure of C-dots were investigated by TEM and the high resolution TEM (HRTEM). TEM image of CDs-180 (Figure 1a) shows that the C-dots are well-dispersed. The particle size distribution of the CDs-180 (Figure 1a inset down) is $1.5-3.5 \mathrm{~nm}$ with an average diameter of $2.5 \mathrm{~nm}$. HRTEM image of CDs-180 (Figure 1a inset up) does not show any clear lattice fringes, indicating their amorphous nature.

The surface structure and components of C-dots were characterized by FTIR and XPS. The FT-IR spectrum of CDs-180 is showed in Figure. 1b. In the figure, there is characteristic absorption broad bands of $\mathrm{O}-\mathrm{H}$ and $\mathrm{N}-\mathrm{H}$ stretching vibration around $3218-3051 \mathrm{~cm}^{-1}[7]$. The peak at $1693 \mathrm{~cm}^{-1}$ is attributed to the $\mathrm{C}=\mathrm{O}$ stretching vibration. The peak at $1563 \mathrm{~cm}^{-1}$ can be identified as the Amide II band $\mathrm{N}-\mathrm{H}$ bond. The peaks at $1401 \mathrm{~cm}^{-1}$ and $1209 \mathrm{~cm}^{-1}$ are considered to be the amine $\mathrm{C}-\mathrm{N}$ bond[8]. XPS survey spectrum of CDs-180 (Figure 1c) shows that they contain three dominant peaks: C1s (around $284.9 \mathrm{eV}$ ), N1s (around $400.3 \mathrm{eV}$ ) and O1s (around $532.8 \mathrm{eV}$ ). In detail, the high-resolution C1s XPS spectrum of CDs-180 (Figure 1d) reveals four peaks with the binding energies at $284.9,285.4,286.9$ and $288.6 \mathrm{eV}$ ascribed to $\mathrm{C}-\mathrm{C}=\mathrm{C}, \mathrm{C}-\mathrm{N}, \mathrm{C}-\mathrm{O}$ and $\mathrm{C}=\mathrm{O}$ species, respectively[9]. 

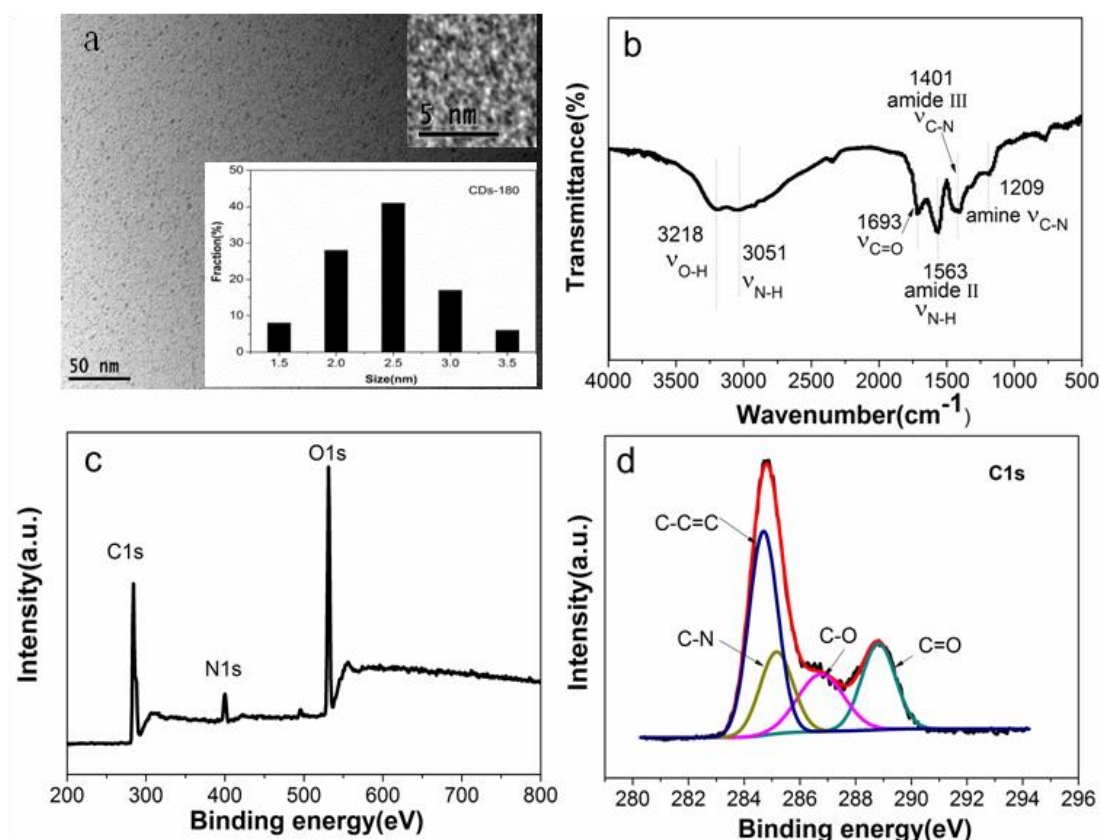

Fig. 1. (a) TEM image, insert up: HRTEM image, insert down: The particle size distribution, (b) FT-IR spectrum, (c) XPS survey spectrum and (d) C1s spectrum of CDs-180.

\subsection{The stability of $C$-dots}

The fluorescent properties under various conditions were further studied. Fluorescent intensity of CDs-180 is not significantly decreased under continuous UV irradiation for 5 hours (Figure 2a), in ambient air at room temperature for 2 months (Figure $2 \mathrm{~b}$ ) and in various concentrations of $\mathrm{NaCl}$ aqueous solution (Figure 2c). Meantime, the PL emission intensity of the C-dots has not too big change at $\mathrm{pH}$ from 2 to 10 (Figure 2d). CDs- 180 shows are outstanding chemical and optical stability, which is helpful for it using as fluorescent probe. 

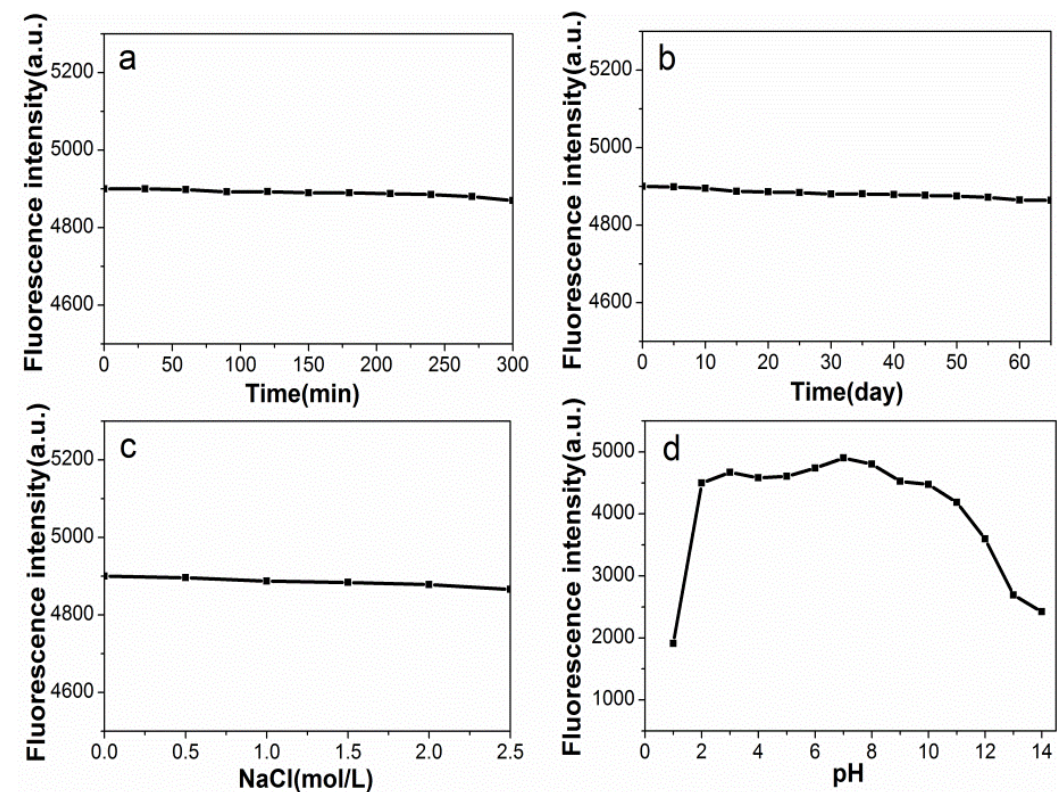

Fig. 2. (a) Effect of time under UV irradiation of $365 \mathrm{~nm}$, (b) effect of time at room temperature, (c) effect of ionic strength (ionic strength were controlled by various concentrations of $\mathrm{NaCl}$ aqueous solution) and (d) effect of $\mathrm{pH}$ (the $\mathrm{pH}$ was adjusted with hydrochloric acid and sodium hydroxide) on fluorescence intensity of CDs- 180 .

\subsection{Optical properties of $\mathrm{C}$-dots}

The photographs of aqueous solution of CDs-180 with good water solubility is shown in Figure 3a (insert). It shows turquoise transparent liquid appearance in daylight while it exhibits blue color fluorescence under the irradiation of $365 \mathrm{~nm}$ ultraviolet (UV) light lamp. Firstly, the ultraviolet-visible (UV-vis) absorption spectrum of CDs-180 (Figure 3a) exhibits characteristic absorption near $200 \mathrm{~nm}$, a very broad absorption band in UV-vis region due to the $\pi \rightarrow \pi^{*}$ transition of aromatic $\mathrm{sp}^{2}$ domains with a tail extending to the visible light region[4]. Furthermore, a very strong and symmetric absorption peak at about $330 \mathrm{~nm}$ can also be observed, which ascribes to the $n \rightarrow \pi^{*}$ transition of $\mathrm{C}=\mathrm{O}[10]$. In the photoluminescence (PL) emission spectra (Figure 3b), CDs-180 displays that emission peak does not change as excitation wavelengths increase. The QY of CDs-180 is as high as $18.95 \%$ in reference to quinine sulfate at $350 \mathrm{~nm}$ excitation, which benefits for serving as a highly sensitive probe. In Figure 3c, CDs-180 present maximum excitation wavelengths at $350 \mathrm{~nm}$ and the maximum emission peak at $440 \mathrm{~nm}$. 

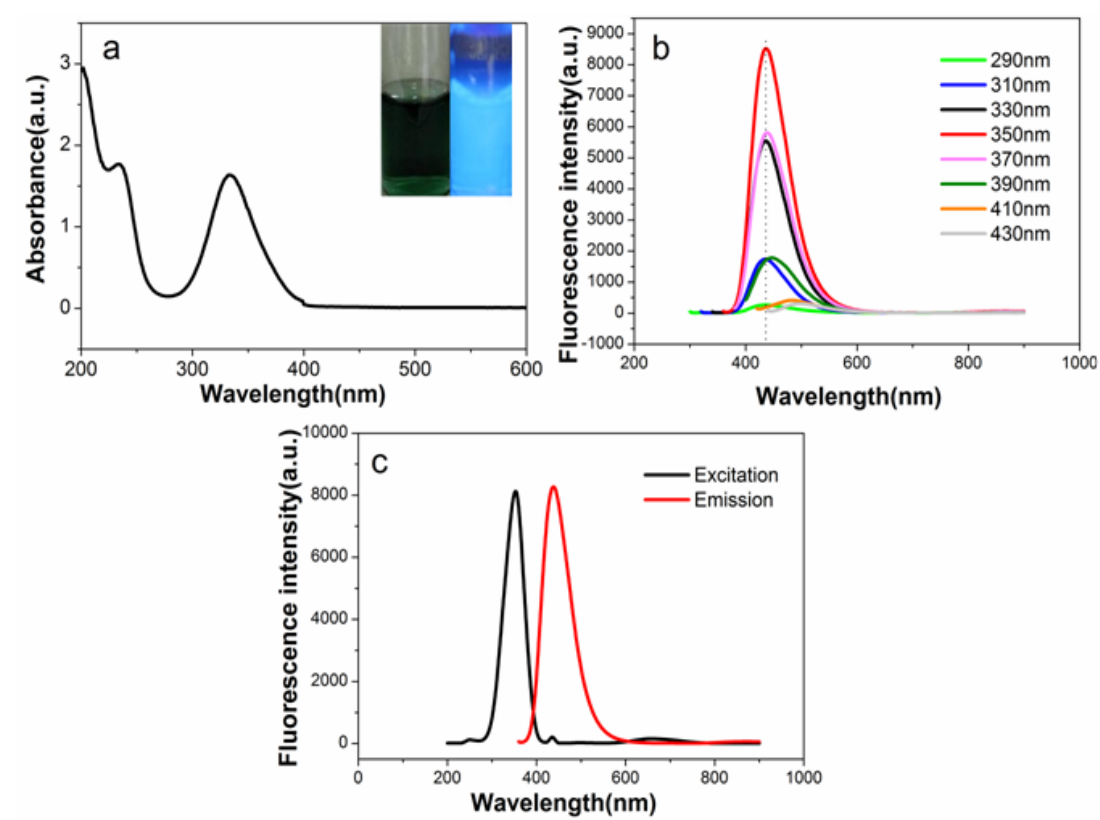

Fig. 3. (a) UV-vis absorption spectrum of CDs-180. (b) PL spectra at different excitation wavelengths of CDs-180. (c) Optimal excitation and emission of PL spectra of CDs- 180.

\subsection{Selectivity and sensitivity of fluorescent probe}

Particularly, the effects of metal ions including $\mathrm{K}^{+}, \mathrm{Na}^{+}, \mathrm{Ca}^{2+}, \mathrm{Mg}^{2+}, \mathrm{Al}^{3+}, \mathrm{Cr}^{3+}$, $\mathrm{Cd}^{2+}, \mathrm{Zn}^{2+}, \mathrm{Ba}^{2+}, \mathrm{Mn}^{2+}$, and $\mathrm{Cr}_{2} \mathrm{O}_{7}{ }^{2-}$ on the fluorescence intensity of C-dots were investigated. Figure. 4a shows that the fluorescence of CDs-180 is not very sensitive to most metal cations (at a concentration of $1.0 \times 10^{-5} \mathrm{M}$ ). However, only $\mathrm{Cr}(\mathrm{VI})$ can effectively quench the fluorescence of CDs-180, suggesting good selectivity of the $\mathrm{C}$-dots for $\mathrm{Cr}(\mathrm{VI})$.

Figure $4 \mathrm{~b}$ shows the fluorescence intensity of CDs-180 in the presence of different concentrations of $\mathrm{Cr}(\mathrm{VI})$ with an excitation at $350 \mathrm{~nm}$. With the increase of $\mathrm{Cr}(\mathrm{VI})$ concentration, CDs-180 fluorescence intensity gradually reduces. When the concentration of $\mathrm{Cr}(\mathrm{VI})$ reached $50 \mu \mathrm{M}$ CDs-180 fluorescence quenching rate achieved $91 \%$. The experiment reveals that the PL intensity of CDs-180 is quenched by $\mathrm{Cr}(\mathrm{VI})$ in the range of 0 to $50 \mu \mathrm{M}$ in a concentration dependent manner which can be well described by the linear regression equations: $10000 / \mathrm{F}=1.80+0.27[\mathrm{C}]$ with a correlation coefficient of 0.9994 (Figure 4c). Here F is PL intensity of CDs-180 and [C] is the $\mathrm{Cr}(\mathrm{VI})$ concentration. The detection limit of $0.015 \mu \mathrm{M}$ is obtained based on a $3 \sigma / \mathrm{k}(\sigma$ is the standard deviation of the blank signal and $\mathrm{k}$ is the slope of the linear 
calibration plot), suggesting that the CDs-180 possesses sensitivity in the detection of $\mathrm{Cr}(\mathrm{VI})$.
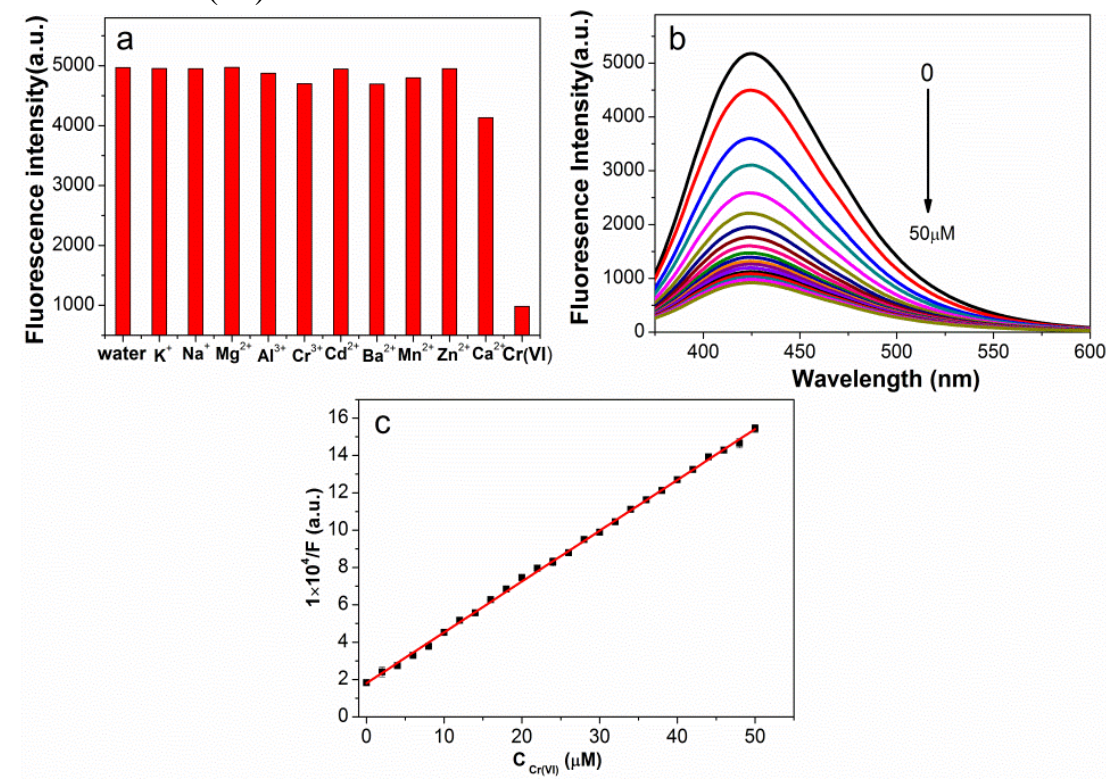

Fig. 4. (a) PL intensity of CDs-180 in the presence of various metal ions aqueous solution ( $\lambda \mathrm{ex}=350$ $\mathrm{nm})$. (b) PL response of CDs-180 in the presence of different concentrations of $\mathrm{Cr}(\mathrm{VI})(\lambda \mathrm{ex}=350$ $\mathrm{nm})$. (c) The plot of PL intensity against $\mathrm{Cr}(\mathrm{VI})$ concentrations ranging from 0 to $50 \mu \mathrm{M}$.

\section{Conclusion}

In summary, we successfully synthesized excitation-independent carbon dots (Cdots) via a facile one-step hydrothermal approach from ammonium citrate,. The as-prepared C-dots show good water-solubility, strong photoluminescence, good photostability, high selectivity and sensitive for $\mathrm{Cr}$ (VI) in a wide concentration range. Due to their excellent properties, these carbon dots were successfully used for $\mathrm{Cr}(\mathrm{VI})$ detection, the detection limitation comes to $0.015 \mu \mathrm{M}$.

\section{References}

1. Li PC, Jiang SJ. Electrothermal vaporization inductively coupled plasmamass spectrometry for the determination of $\mathrm{Cr}, \mathrm{Cu}, \mathrm{Cd}, \mathrm{Hg}$ and $\mathrm{Pb}$ in rice flour. Anal Chim Acta. 2003;495:143-150 .

2. Madrakian T, Haghshenas E, Afkhami A. Simultaneous determination of tyrosine, acetaminophen and ascorbic acid using gold nanoparticles/multiwalled carbon nanotube/glassy carbon electrode by differential pulse voltammetric method. Sens Actuators B.2014; 193:451460 . 
3. Khosravi F, Asadollahzadeh H Determination of ascorbic acid in different citrus fruits under reversed phase conditions with UPLC. Euro J Exp Biol.2014; 4:91-94.

4. Sun Y-P, Zhou B, Lin Y, et al. Quantum-Sized Carbon Dots for Bright and Colorful Photoluminescence. Journal of the American Chemical Society. 2006;128(24):7756-7757.

5. Zheng L, Chi Y, et al. Electrochemiluminescence of Water-Soluble Carbon Nanocrystals Released Electrochemically from Graphite. Journal of the American Chemical Society. 2009;131(13):4564-4565.

6. Baker SN, Baker GA. Lumineszierende Kohlenstoff-Nanopunkte: Nanolichtquellen mit Zukunft. Angewandte Chemie. 2010;122(38):68766896.

7. Jiang J, He Y, Li S, Cui H. Amino acids as the source for producing carbon nanodots: microwave assisted one-step synthesis, intrinsic photoluminescence property and intense chemiluminescence enhancement. Chemical Communications. 2012;48(77):9634-9636.

8. Zhi Y, Minghan X, Yun L, et al. Nitrogen-doped, carbon-rich, highly photoluminescent carbon dots from ammonium citrate. Nanoscale. 2014;6(3):1890-1895.

9. Bourlinos AB, Stassinopoulos A, Anglos D, et al. Surface Functionalized Carbogenic Quantum Dots. Small. 2008;4(4):455-458.

10. Nie H, Li M, Li Q, et al. Carbon Dots with Continuously Tunable FullColor Emission and Their Application in Ratiometric $\mathrm{pH}$ Sensing. Chemistry of Materials. 2014;26(10):3104-3112. 